\title{
Effects of Probiotic Mixture Supplementation and Evaluation of Intestinal Mucosal Tolerance and Gut Microbiome in Newborns with Perinatal Asphyxia Receiving Hypothermic Treatment: A Randomized, Multicentric, Blinded, Controlled TRIAL (VISNAT)
}

Maria Elisabetta Baldassarre

University of Bari "Aldo Moro"

\section{Raffaella Panza}

University of Bari "Aldo Moro"

Antonio Di Mauro ( $\square$ antonio.dimauro@uniba.it )

University of Bari "Aldo Moro" https://orcid.org/0000-0001-7052-9784

Margherita Fanelli

University of Bari "Aldo Moro"

Paola Mastromarino

Universita degli Studi di Roma La Sapienza

Salvatore Scacco

University of Bari "Aldo Moro"

Manuela Capozza

University of Bari "Aldo Moro"

Donatella Capodiferro

University of Bari "Aldo Moro"

Nicola Laforgia

University of Bari "Aldo Moro"

Study protocol

Keywords: Asphyxia neonatorum [MeSH], Probiotics [MeSH], Infant, Newborn [MeSH], neurodevelopment.

Posted Date: June 28th, 2021

DOI: https://doi.org/10.21203/rs.3.rs-266824/v1

License: (c) (i) This work is licensed under a Creative Commons Attribution 4.0 International License.

Read Full License 


\section{Abstract}

\section{Introduction}

Neonatal encephalopathy is still a major cause of mortality and morbidity for newborns, although hypothermia treatment has improved the prognosis of term newborns with hypoxic-ischemic injury.

Recent evidence suggests that one of the crucial but understudied mechanisms of secondary neuronal injury after global hypoxic-ischemic injury is inflammation.

Hence, blocking the inflammatory reaction promotes neuroprotection and has a potential for use in the clinical treatment of ischemic brain injury.

Many preclinical studies have shown bidirectional interactions between the central nervous system, the enteric nervous system, and the gastrointestinal tract, suggesting a prominent role for the gut microbiota in these gut-brain interactions. Early human studies suggest that altering the microbiota with beneficial bacteria, or probiotics, can lead to changes in brain function, as well as subjective reports of mood.

\section{Methods and analysis}

The VISNAT trial is a PILOT STUDY developed according to SPIRIT checklist. It is a randomized, placebocontrolled, blinded, multicentre superiority trial with two parallel groups and a primary outcome of mortality and/or disability at 18 months of age.

The experimental intervention will consist in administrating a high-dose multi-strain probiotic (SIVOMIXX'M) for 30 days in addition to hypothermic therapy.

Primary outcome will be to compare mortality and/or disability at 18 month of age between the two groups. Disability will be assessed by means of the Bayley Mental Development Index. Secondary outcomes will be to compare inflammatory cytokines level and brain injury biomarkers (i.e. Tau and neurofilament light protein) between groups.

A subgroup analysis will be performed stratifying patients according to both probiotic/placebo treatment and breast/artificial milk.

\section{Discussion}

Probiotics supplementation represents a simple and reproducible intervention. If proven effective, probiotics supplementation in asphyxiated babies would improve clinical outcomes and reduce health cost. Hence, this study may cast a new light on the management of hypoxic-ischemic encephalopathy.

\section{Trial registration number}

Study has been registered on clinicaltrials.org on $23^{\text {rd }}$ October 2019 with number NCT04145713. 


\section{Trial Status}

The present trial (VISNAT Study Version 1.0 - 23 October 2019) has been approved by the Ethics Committees of the coordinator centre "Azienda Ospedaliera Universitaria Consorziale Policlinico di Bari". Approval from the Ethics Committees of each participating hospital has also been requested. Enrollment of patients is planned to start in July 2021 and should last approximately 2 years.

\section{Introduction}

\section{Research Question}

In newborns with perinatal asphyxia receiving hypothermic treatment, might the add-on of high-dose of multi-strain probiotic (SIVOMIXX ${ }^{\mathrm{TM}}$ ) reduce mortality and/or disability at 18 months of age versus placebo?

\section{Background}

Neonatal encephalopathy is still a major cause of mortality and morbidity for newborns, although hypothermia treatment has improved the prognosis of term newborns with hypoxic-ischemic $(\mathrm{HI})$ injury. ${ }^{1}$

Long-term neurological sequelae still develop in approximately $65 \%$ of all the asphyxiated newborns treated with hypothermia, yet an improvement compared to $78 \%$ neurodevelopmental morbidity in the non-treated newborns. ${ }^{2}$

Recent evidence suggests that a cascade of cellular and biochemical responses to the initial $\mathrm{HI}$ insult can lead to secondary neuronal injury after re-oxygenation. ${ }^{3}$

One of the crucial but understudied mechanisms of secondary neuronal injury after global $\mathrm{HI}$ is inflammation which is characterized by activation of microglia, the innate immune cells of brain; ${ }^{4}$ migration of peripheral macrophages; ${ }^{5}$ release of proinflammatory cytokines and chemokines, and phagocytosis of injured and uninjured neurons. ${ }^{6}$

Some evidence suggests that blocking the inflammatory reaction promotes neuroprotection and has a potential for use in the clinical treatment of ischemic brain injury. ${ }^{3}$

In this regard, it should be stressed that many preclinical studies have shown bidirectional interactions between the central nervous system, the enteric nervous system, and the gastrointestinal tract, suggesting a prominent role for the gut microbiota in these gut-brain interactions. ${ }^{7}$ Current evidence suggests that multiple mechanisms, including immune, endocrine and neurocrine pathways, may be involved in gut microbiota-to-brain signalling and that the brain can in turn alter microbial composition and behaviour via the autonomic nervous system. ${ }^{8}$ 
Early human studies suggest that altering the microbiota with probiotics, can lead to short- and long-term health benefits. In particular, probiotics have being increasingly scrutinized as prophylactic ${ }^{9}$ and therapeutical tools ${ }^{10}$ to reduce the socio-economic burden of functional gastrointestinal diseases, ${ }^{11,12} \mathrm{a}$ model of disorders of Gut-Brain Interaction in children. ${ }^{13}$ Authors have speculated that probiotics supplementation affects release of immunity-modulating molecules, with consequent health benefits related to the gut microbiota-to-brain signalling.

There are increased chemokine gene expression and release in the developing brain after $\mathrm{HI}$, some investigators have measured serum cytokines in newborns that received hypothermic treatment, showing a distinctive pattern of chemokines in the acute phase of injury. In particular, increased levels of serum interleukin (IL)- 6 and IL-8 have been found in the first 24-48 hours after birth. ${ }^{14,15}$ Jenkins et al. have also demonstrated that some cytokines, such as IL-6, show a biphasic pattern, connected with the theory of cytokine-mediated repair at later time points. ${ }^{14}$

Moreover, several authors have focused on the correlation between neonatal serum cytokine levels, magnetic resonance imaging (MRI) abnormalities and neurodevelopmental outcome at 24 or 30 months of age.

Bartha et al. have found a significant association between IL-13, IL-6, IL-8 and TNF-a serum levels, high lactate/choline ratio in the deep grey nuclei (DGN) assessed by proton magnetic resonance spectroscopy (MRS) and abnormal neurodevelopmental outcome at 30 months of age scored using Bayley Scales of Infant Development II test. ${ }^{16}$

Furthermore, recent studies have confirmed that diffusion-weighted imaging (DWI) is the most reliable MRI sequence to assess injury during the first week after an $\mathrm{HI}$ event, specially to estimate the injury to the deep grey matter area that has the most predictive property in prognosticating outcome. Early detection is important for the selection of future additional neuroprotective strategies, which may need to be initiated within the first week after birth. For these reasons some investigators have developed a novel easily applicable score based on the assessment of all MRI abnormalities of suspected importance for prognostication in infants with $\mathrm{HI}$ encephalopathy; this kind of score system has the advantage of including DWI, in contrast to most of the previously published MRI score system, such as Barkovich scoring system, that were designed to be performed using T1- and T2-weighted images only and therefore use scans obtained in the second week of life. ${ }^{17}$

Considering that none of these studies have taken into account the effects of probiotics on neonates with asphyxia, our group intends to conduct a pilot study that compares the add-on of SIVOMIXX ${ }^{\mathrm{TM}}$ probiotic over the baseline therapy (hypothermia) versus placebo.

According to the Italian national guidelines on hypothermia, babies are kept nil by mouth throughout the duration of the hypothermic treatment. ${ }^{18}$ 
Thus far, there is no evidence available regarding whether the type of milk started right after the discontinuation of the hypothermic treatment might influence the patient feeding tolerance. Therefore, we also aim to assess the impact of different types of milk (breast vs artificial milk) on gut microbiome and time to reach full feeds in asphyxiated babies after hypothermic treatment.

\section{Methods And Analysis}

\section{Study Design}

The VISNAT trial is a PILOT STUDY due to absence of previous evidences in literature on using probiotic in newborns with perinatal asphyxia. It has been developed according to SPIRIT checklist (Supplementary 1). It is designed as a randomized, placebo-controlled, blinded, multicentre superiority trial with two parallel groups and a primary outcome of mortality and/or disability at 18 months of age. After informed consent (Supplementary 2) is obtained from both parents, randomization will be performed as block randomization with a 1:1 allocation using a computer-generated allocation sequence, while the allocation concealment will be performed using locked bags. Randomization data and allocation list will be stored in a secure place and will not be available to any of the components of the study apart of data collectors.

Participant files will be stored for a period of 10 years after completion of the study. All the components of the study will be blinded including: participants and their parents, healthcare providers, outcome assessors, data collectors, data analysts.

\section{Patient and Public Involvement}

Patient and Public Involvement was not sought in the design of this study.

\section{Participants: inclusion/exclusion criteria}

Inclusion criteria:

- Newborns with gestational age $>/=35$ weeks and birth weight $>/=1800$ grams

- Intrapartum asphyxia defined according at least one of the following:

- APGAR index at 10 minutes $</=5$;

- Resuscitation with endotracheal tube or mask IPPV for more than 10 minutes;

- $\mathrm{pH}</=7$ or excess of base $>/=12 \mathrm{mmol} / \mathrm{l}$ on arterial blood gas $(\mathrm{ABG})$ analysis within the first 60 minutes of life

- Moderate/severe Hypoxic-ischemic encephalopathy assessed between 30 and 60 minutes of life according to Sarnat \& Sarnat definition

- Hypothermic treatment. According to the Italian national guidelines on Perinatal Asphyxia and Therapeutic Hypothermia, the hypothermic therapy will be started in the first six hours of life and will last 72 hours. 


\section{Exclusion criteria:}

- Inability to obtain the informed consent from both parents

- Congenital major malformations or syndromes

- Surgical diseases

No concomitant care and interventions are prohibited during the trial.

\section{Setting and recruitment}

In order to reach the prespecified sample size (see below 13. Statistical Issue: hypothesis, sample size and power) the VISNAT trial will involve 9 centres:

1. Azienda Ospedaliera Universitaria Consorziale Policlinico di Bari (mariaelisabetta.baldassarre@uniba.it) (coordinator centre)

2. Azienda Ospedaliera Regionale San Carlo di Potenza (Camilla Gizzi; camillagizzi@tin.it)

3. Azienda Ospedaliera “V. Fazzi” di Lecce (Giuseppe Presta; gpresta@alice.it)

4. Azienda Ospedaliera "A. Perrino" di Brindisi (Enrico Rosati)

5. Azienda Ospedaliera Universitaria di Foggia - Ospedali Riuniti (Giuseppe Popolo; gpopolo@ospedaliriunitifoggia.it)

6. Azienda Ospedaliera Universitaria "Federico II" di Napoli (Maria Vendemmia)

7. Azienda Ospedaliera Universitaria di Modena (Licia Lugli)

8. Azienda Ospedaliera Universitaria “Sant'Anna” di Ferrara (Elisa Ballardini)

9. Azienda Ospedaliera "Bolognini" di Seriate (Felicia Varsalone)

Each clinical centre involved in the VISNAT was chosen based on documentation for patient availability.

The coordinator centre will be the Neonatal Intensive Care Unit "A.Mautone" of Policlinico di Bari. It is an academic department specialized in the care of newborns at risk due to several conditions such as extreme prematurity, surgical and cardiac diseases, metabolic diseases, neurological diseases and perinatal asphyxia treated with systemic hypothermia. Our research team is made of neonatologists with considerable experience in intensive care, neonatal gastroenterology and neurology, supported by a group of residents and young paediatricians constantly involved in research and clinical trials. Our team also actively collaborates with other academic units, as well as, Neuroradiology unit for brain MRI in asphyxiated infants treated with systemic hypothermia and Laboratory unit for all blood chemistry tests. During time we have performed many research protocols with scientific papers published in peer-reviewed scientific journal.

Each of the others centres has a Neonatal Intensive Care Unit and has been chosen based on patient recruitment capability. 
After obtaining the informed consent, the healthcare providers will enrol the patients that meet the inclusion criteria and proceed to randomization. All the interventions will take place in the Neonatal Intensive Care Units.

\section{Timeframe}

The recruitment period will extend over 2 years, while the follow-up will last up to 2 years.

\section{Intervention}

The experimental intervention will consist in administrating a high-dose multi-strain probiotic (SIVOMIXX'M) for 30 days in addition to hypothermic therapy. The administration of probiotics will begin during the first 24 hours of hypothermia, at the dose of 1 capsule open in 5 millilitre of water. It will be administered daily for 30 days, either via nasogastric tube or orally.

\section{Comparison}

The comparison intervention will consist in administrating placebo for 30 days in addition to hypothermic therapy. The administration of placebo will begin during the first 24 hours of hypothermia, at the dose of 1 capsule open in 5 millilitre of water. It will be administered daily for 30 days, either via nasogastric tube or orally.

\section{Type of milk}

Newborns will not be randomized for type of milk. Mothers will be encouraged to give their own breast milk. In case of unavailability of breast milk, all enrolled newborns will be fed with the same artificial milk.

The type of milk (breast/artificial) will be registered in clinical notes.

\section{Clinical assessment, laboratory tests and brain imaging (Supplementary 3)}

1. Weekly auxological data (weight, height, head circumference) will be recorded during hospitalisation. Upon discharge, enrolled patients will be followed up in the outpatient service with periodical clinical evaluation.

Bayley Mental Development Index (MDI) will be performed at 18 months to assess the degree of disability (no disability, mild, moderate or severe disability).

2. Blood sample ( $3 \mathrm{ml})$ for inflammatory cytokines levels (IL-1 $1 \beta, \mathrm{IL}-6$, IL-8 and TNF-a) will be collected within 24 hours of life and then daily throughout the 72-hour hypothermic treatment and at the end of probiotic supplementation (day 30). The analysis of the samples will be performed by ELISA assay at the coordinator centre (Dr. Thea Magrone - Department of Basic Medical Sciences, Neurosciences and Sensory Organs, A.O.U. Policlinico of Bari). Blood sample (3 ml) for Tau and 
neurofilament light protein levels will be collected within 24 hours of life, daily during hypothermia and at the end of the intervention (day 30) and analysed by ELISA assay at the coordinator centre (Prof. Salvatore Scacco - Department of Basic Medical Sciences, Neurosciences and Sensory Organs, A.O.U. Policlinico of Bari).

A stool sample will be collected for microbiome analysis in every enrolled newborn at the end of hypothermic treatment (i.e. before the beginning of oral feeding) and at the end of probiotic treatment. The analysis of the samples will be performed by Real-time PCR at the Department of Microbiology - University “La Sapienza”, Rome (Prof. Paola Mastromarino).

3. According to the Italian national guidelines on neonatal asphyxia and therapeutic hypothermia, brain MRI will be scheduled at the end of the first week of life and during the second month of life.

\section{Outcomes}

Primary outcome will be to compare mortality and/or disability at 18 month of age between the two groups.

Disability will be assessed by means of the Bayley MDI.

Severe disability will be defined by any of the following: Bayley MDI < 70; Gross Motor Function (GMF) level of 3-5; blindness or profound hearing loss requiring amplification.

Moderate disability will be defined as Bayley MDI between 70-84 and any of the following: GMF level = 2, a seizure disorder or a hearing deficit.

Mild disability will be defined by either Bayley MDI of 70-84 alone or a MDI > 85 and any of the following: $\mathrm{GMF}$ level $=2$, seizure disorder or hearing loss not requiring amplification.

Infants with Bayley MDI > 85, GMF level = 1, absence of any neurosensory deficits will be defined as "no disability".

Secondary outcomes will be to compare inflammatory cytokines level and brain injury biomarkers (i.e. Tau and neurofilament light protein) between groups.

A subgroup analysis will be performed stratifying patients according to both probiotic/placebo treatment and breast/artificial milk. The aim will be to assess changes in gut microbiota and compare oral tolerance, the latter being defined as the number of days to reach full enteral feeding $(140 \mathrm{ml} / \mathrm{kg})$, in each subgroup.

\section{Statistical Issue: hypothesis, sample size and power}

This is prospective superiority study. The sample size, due to absence of previous evidences in literature on using probiotic in newborns with perinatal asphyxia, was established on the bases of TOBY's results in which $45 \%$ of mortality and/or disability in the group with hypothermic therapy was observed. ${ }^{19}$ 
Hypotizing a $30 \%$ (with a superiority margin of $10 \%$ ) of reduction in the proportion of mortality and/or disability in the group with a high-dose multi-strain probiotic in addition to hypothermic therapy respect to the hypothermic treatment group, a power $1-b=0.80$ and a significant level $a=0.05$, a sample of 55 newborns for each group are needed. Considering a $10 \%$ of dropout we decided to enrol 120 newborns over a two-year period (60 probiotics, 60 placebo). ${ }^{20}$

Statistical analysis will be performed by Prof. Margherita Fanelli.

The primary outcome will be evaluated comparing proportions of mortality and/or disability by means of $z$ test. The effect of probiotics on the primary outcome will be evaluated adjusting for the centre and some clinically relevant variables (gestational age, birth weight) by means of logistic regression model. A survival analysis will be performed by Kaplan Meyer Overall Survival and Free Survival curves that will be compared by Log Rank test. Cox regression models will be built to evaluate the effects of probiotic and other clinically variables on the survival time.

The secondary outcomes will be analysed as follows: binary outcomes will be compared between the two arms using Chi-square test, inflammatory cytokines level and brain injury biomarkers will be compared between the two arms using Student $t$ test or Mann-Whitney test, as appropriate while number of days to reach full feeds will be evaluated by Kaplan Meyer curves compared between the two arms by log rank test. Logistic regression models will be built to evaluate the effect of probiotics on the binary outcomes, adjusting for the centre and some clinically relevant variables (gestational age, birth weight, Sarnat).

A p-value less than 0.05 will be considered statistically significant. Statistical analysis will be performed using SPSS vers. 21.

\section{Ethics And Dissemination}

\section{Compliance to protocol}

Compliance will be defined as full adherence to protocol. Compliance with the protocol will be ensured by the local PI and collaborators in each hospital; they will be responsible for local data collection.

SIVOMIXX' ${ }^{\text {TIM }}$ packages of each study will be collected be the investigators at the end of treatment to assess adherence. The local PI will weekly monitor the adherence to the study protocol and will input the patients' data in an electronic data sheet. Double data entry will be performed by 2 independent members at each centre to promote data quality.

\section{Missing data}

Once an infant is enrolled or randomized, the study site will make every reasonable effort to follow the infant for the entire study period. It is projected that the rate of loss-to-follow-up on an annual basis will be at most $5 \%$. Study site staff are responsible for developing and implementing local standard operating procedures to achieve this level of follow-up. 
Investigators and study staff will be trained on the importance of the completion of the study period of enrolled patients. Parents will also be informed about this crucial aspect to reduce dropout, and a local investigator will be available at each site anytime the parents may need further information or clarification during the study period.

\section{Data Safety and Monitoring Board}

Safety measures will include incidence, severity and causality of reported serious adverse events (SAEs), represented by changes in occurrence of the expected common neonatal complications and the development of unexpected SAEs. During systemic hypothermia the protocol to control temperature, metabolic status, haematological status, infectious disease will be applied.

SAE will be defined as unexpected death, sepsis and enterocolitis. All SAEs will be followed until complete resolution or until the clinician responsible for the care of the recruited patient considers the event to be chronic or the infant to be stable. Any adverse event will be reported to the Principal Investigator Centre.

A monitoring board including an independent assessor (not involved in the study) from the Azienda Ospedaliera Universitaria Consorziale Policlinico di Bari and assessors from each participating hospital will review all the deaths and adverse effects. If there is a reasonable suspected causal relationship with the intervention, SAEs will be reported to the Ethics Committee to guarantee the safety of the participants. The intervention will be discontinued if any of the following occur: parents' consent withdrawal, attending physician decision or occurrence of serious adverse event possibly related to probiotic supplementation.

To maintain the overall quality and legitimacy of the clinical trial, code breaks should occur only in exceptional circumstances when knowledge of the actual treatment is absolutely essential for further management of the patient. Investigators are encouraged to discuss with the Medical Advisor if unblinding is necessary.

If unblinding is deemed to be necessary, the investigator should use the system for emergency unblinding through local emergency number.

The Investigator is encouraged to maintain the blind as far as possible. The actual allocation must NOT be disclosed to the patient and/or other study personnel including other site personnel, monitors, corporate sponsors or project office staff; nor should there be any written or verbal disclosure of the code in any of the corresponding patient documents.

The Investigator must report all code breaks (with reason) as they occur on the corresponding CRF (case report form) page.

Unblinding should not necessarily be a reason for study drug discontinuation.

An interim analysis will be performed on the primary endpoint and SAEs from the first 16 infants enrolled. The interim analysis will be performed by the statistician, blinded for the treatment allocation, who will 
report to the principal investigator. The principal investigator will discuss the results of the interim analysis with the monitoring board and the trial will be ended in case of harm. Criteria for stopping for harm include: a statistically significant difference in the primary outcome between the treatment groups; a reasonable suspected causal relationship between the intervention and SAEs.

\section{Confidentiality}

Only local PI will have access to the electronic database with an assigned personal account and password. Subjects will be identified by sex, birth date, and assigned trial number, during and after the trial, in accordance with personal data protection law.

\section{Access to data}

The principal investigators of each site will have complete access to the final trial dataset, and no contractual agreement exists to limit such access for the investigators.

\section{Consent/assent}

Sub-investigators will introduce the trial to patients. Patients will also receive information sheets. Patients will then be able to have an informed discussion with the sub-investigators, who will obtain written consent from patients willing to participate in the trial. Information sheets and consent forms are provided for all parents involved in the trial.

\section{Other issue (registration, sponsor etc.)}

The VISNA trial will involve all the parts cooperatively. A preliminary meeting intended to clarify the design, methods and outcomes of the study will be the first step of the protocol. Teaching materials will be designed, realised and distributed to all the members of the research team. Monthly meetings will be planned to discuss any aspects of the protocol. Training sessions will take place in the teaching room of Neonatal Intensive Care Unit "A. Mautone" of Policlinico di Bari and will be chaired by the Principal Investigator together with the collaboration of co-investigator.

Study has been registered on clinicaltrials.org on 23rd October 2019 with number NCT04145713.

The Investigational Product (probiotic mixture) and placebo will be supplied by Ormendes SA.

\section{Strengths And Limitations Of This Study}

- This study comprises a well-powered clinical trial investigating the additional effect of probiotics on the neurodevelopmental outcome of asphyxiated neonates treated with hypothermia.

- A strength of the trial is the pragmatic nature of the study and applicability in daily clinical practice.

- The multicentric nature of the study enables to assess the reproducibility of the intervention. 


\section{Study sponsor}

Ormendes SA, 24 Chemin des vignettes 1008 Jouxtens-Mézery - CH

Study sponsor will be accountable of the supply of the Investigational Product and the placebo. Study sponsor has no role in study design, collection, management, analysis, and interpretation of data, writing of the report, and the decision to submit the report for publication.

\section{Organisational structure and responsibilities}

\section{Principal Investigator and Research Physician}

Design and conduct of VISNAT

Preparation of protocol and revisions

Preparation of investigators brochure (IB) and CRFs [Case Report Forms]

Organising steering committee meetings

Managing CTO [Clinical Trials Office]

Publication of study reports

Members of TMC [Trial Management Committee]

\section{Steering committee (SC)}

Agreement of final protocol

All lead investigators will be steering committee members

Recruitment of patients and liasing with principal investigator

Reviewing progress of study and if necessary agreeing changes to the protocol and/or investigators brochure to facilitate the smooth running of the study.

\section{Trial Management Committee (TMC)}

Encompasses Principal investigator, Research Physician, and Administrator

Study planning

Organisation of steering committee meetings

Provide annual risk report and ethics committee 
Reports Serious unexpected suspected adverse events

Responsible for trial master file

Advice for lead investigators

Audit of 6 monthly feedback forms via emails and group calls from Coordinator Centre

Assistance with international review, board/independent ethics committee applications

Data verification

Randomisation

Organisation of central serum sample collection

\section{Data Manager}

Maintenance of trial IT system and data entry

Data verification

\section{Lead Investigators}

In each participating centre a lead investigator (senior neonatologist) will be identified, to be responsible for identification, recruitment, data collection and completion of CRFs, along with follow up of study patients and adherence to study protocol and investigators brochure. Lead investigators will be steering committee members.

\section{Abbreviations}

ABG - arterial blood gas; AOU - Azienda Ospedaliera Universitaria; AOUC - Azienda Ospedaliera Universitaria Consorziale; CRFs - Case Report Forms; CTO - Clinical Trials Office; DGN - deep grey nuclei; DWI - diffusion-weighted imaging; EC - Ethics Committee; GCP - Good Clinical Practice; GMF - Gross Motor Function; HI - hypoxic-ischemic; IL - interleukin; IB - investigators brochure; IPPV - Intermittent Positive Pressure Ventilation; MDI - Mental Development Index; MRI - magnetic resonance imaging; MRS magnetic resonance spectroscopy; PI - Principal Investigator; SAEs - serious adverse events; SC - Steering committee; TMC - Trial Management Committee

\section{Declarations}

\section{Ethics approval and consent to participate}

This study will be conducted in accordance with the ethical principles that have their origin in the Declaration of Helsinki and will be consistent with Good Clinical Practice (GCP) and applicable regulatory 
requirements.

The study will be conducted in compliance with the protocol. The protocol and any amendments and the informed consent will receive Ethics Committee (EC) approval/favourable opinion prior to initiation.

Freely given written informed consent must be obtained from every participant's parent or legally authorized representative prior to clinical trial participation.

The rights, safety, and well-being of the trial participants are the most important consideration and should prevail over interests of science and society.

Patients that are enrolled into the study are covered by local insurance policies.

Study personnel involved in conducting this trial will be qualified by education, training, and experience to perform their respective task(s).

The trial has been approved by the Ethics Committees of the coordinator centre "Azienda Ospedaliera Universitaria Consorziale Policlinico di Bari" (Supplementary 4) and will be sent to the Ethics Committees of each participating hospital. We have planned to start enrollment of patients in July 2021.

Any modifications to the protocol which may impact on the conduct of the study, potential benefit of the patient or may affect patient safety, including changes of study objectives, study design, patient population, sample sizes, study procedures, or significant administrative aspects will require a formal amendment to the protocol. Such amendment will be agreed upon by all Principal Investigators, and approved by the Ethics Committee prior to implementation.

\section{Consent for publication}

The results of the trial are expected to be published in a scientific journal and to be presented in medical seminars and conferences. The final reporting will follow the CONSORT Report guidelines (http://www.consort-statement.org).

The VISNAT Participant Box will list all professionals that have participated in the VISNAT study for a minimum of one year.

\section{Availability of data and materials}

The data that support the finding of this study will be available from the PI (M.E.B.) upon reasonable request.

\section{Competing interests}

The authors declare no conflict of interest.

\section{Funding statement}


This work was supported by Mellin S.p.A that funded one PhD fellowship for the realization of this project. We also thank ORMENDES SA for the supply of study product and placebo.

Either company has no role in the design of the study and collection, analysis, and interpretation of data and in writing the manuscript.

\section{Authors' Contributions}

M.E.B. (Principal Investigator) and N.L. conceptualized and designed the study; she will lead data acquisition, analysis, and interpretation. She will have full access to all of the data in the study and takes responsibility for the integrity of the data and the accuracy of the data analysis. R.P., A.D.M. and M.C. made substantial contributions to study design. They will enrol participants and assign participants to interventions. They will also be responsible for acquisition of data. M.F. is in charge of the statistical aspects (generation of allocation sequence and data analysis). P.M. will perform microbiological and cytokine analysis. S.S. will perform ELISA assay for Tau and neurofilament light protein. The final version of the protocol was seen and finally approved by all authors as submitted.

\section{Acknowledgments}

We thank our colleagues and nurses in each of the 9 participating hospitals who gave their support to this project.

We thank in particular the VISNAT Study Group that encompasses the responsible physicians and investigators of each centre: Camilla Gizzi; Giuseppe Presta; Enrico Rosati; Giuseppe Popolo; Maria Vendemmia; Licia Lugli; Elisa Ballardini and Felicia Varsalone.

\section{References}

1. Schump EA. Neonatal Encephalopathy: Current Management and Future Trends. Crit Care Nurs Clin North Am. 2018;30(4):509-21. doi:10.1016/J.CNC.2018.07.007.

2. Al Amrani F, Marcovitz J, Sanon P-N, et al. Prediction of outcome in asphyxiated newborns treated with hypothermia: Is a MRI scoring system described before the cooling era still useful? Eur $\mathrm{J}$ Paediatr Neurol. 2018;22(3):387-95. doi:10.1016/J.EJPN.2018.01.017.

3. Bhalala US, Koehler RC, Kannan S. Neuroinflammation and Neuroimmune Dysregulation after Acute Hypoxic-Ischemic Injury of Developing Brain. Front Pediatr. 2015;2:144. doi:10.3389/fped.2014.00144.

4. Mallard C, Tremblay M-E, Vexler ZS. Microglia and Neonatal Brain Injury. Neuroscience. 2019;405:68-76. doi:10.1016/j.neuroscience.2018.01.023.

5. Ziemka-Nalecz M, Jaworska J, Zalewska T. Insights Into the Neuroinflammatory Responses After Neonatal Hypoxia-Ischemia. J Neuropathol Exp Neurol. 2017;76(8):644-54. doi:10.1093/jnen/nlx046. 
6. Liu F, Mccullough LD. Inflammatory responses in hypoxic ischemic encephalopathy. Acta Pharmacol Sin. 2013;34(9):1121-30. doi:10.1038/aps.2013.89.

7. Indrio F, Riezzo G, Raimondi F, Di Mauro A, Francavilla R. Microbiota Involvement in the Gut-Brain Axis. J Pediatr Gastroenterol Nutr. 2013;57:11-5. doi:10.1097/01.mpg.0000441927.20931.d6.

8. Tillisch K. The effects of gut microbiota on CNS function in humans. Gut Microbes. 2014;5(3):40410. doi:10.4161/gmic.29232.

9. Baldassarre M, Di Mauro A, Mastromarino P, et al. Administration of a Multi-Strain Probiotic Product to Women in the Perinatal Period Differentially Affects the Breast Milk Cytokine Profile and May Have Beneficial Effects on Neonatal Gastrointestinal Functional Symptoms. A Randomized Clinical Trial. Nutrients. 2016;8(11):677. doi:10.3390/nu8110677.

10. Baldassarre M, Di Mauro A, Tafuri S, et al. Effectiveness and Safety of a Probiotic-Mixture for the Treatment of Infantile Colic: A Double-Blind, Randomized, Placebo-Controlled Clinical Trial with Fecal Real-Time PCR and NMR-Based Metabolomics Analysis. Nutrients. 2018;10(2):195. doi:10.3390/nu10020195.

11. Indrio F, Di Mauro A, Riezzo G, Cavallo L, Francavilla R. Infantile colic, regurgitation, and constipation: an early traumatic insult in the development of functional gastrointestinal disorders in children? Eur J Pediatr. 2015;174(6):841-2. doi:10.1007/s00431-014-2467-3.

12. Indrio F, Di Mauro A, Di Mauro A, et al. Prevention of functional gastrointestinal disorders in neonates: clinical and socioeconomic impact. Benef Microbes. 2015;6(2):195-8. doi:10.3920/BM2014.0078.

13. Drossman DA, Hasler WL. Rome IV-Functional GI Disorders: Disorders of Gut-Brain Interaction. Gastroenterology. 2016;150(6):1257-61. doi:10.1053/j.gastro.2016.03.035.

14. Jenkins DD, Rollins LG, Perkel JK, et al. Serum Cytokines in a Clinical Trial of Hypothermia for Neonatal Hypoxic-Ischemic Encephalopathy. J Cereb Blood Flow Metab. 2012;32(10):1888-96. doi:10.1038/jcbfm.2012.83.

15. Chalak LF, Sánchez PJ, Adams-Huet B, Laptook AR, Heyne RJ, Rosenfeld CR. Biomarkers for Severity of Neonatal Hypoxic-Ischemic Encephalopathy and Outcomes in Newborns Receiving Hypothermia Therapy. J Pediatr. 2014;164(3):468-74.e1. doi:10.1016/J.JPEDS.2013.10.067.

16. Bartha Al, Foster-Barber A, Miller SP, et al. Neonatal Encephalopathy: Association of Cytokines with MR Spectroscopy and Outcome. Pediatr Res. 2004;56(6):960-6. doi:10.1203/01.PDR.0000144819.45689.BB.

17. Weeke LC, Groenendaal F, Mudigonda K, et al. A Novel Magnetic Resonance Imaging Score Predicts Neurodevelopmental Outcome After Perinatal Asphyxia and Therapeutic Hypothermia. J Pediatr. 2018;192:33-40.e2. doi:10.1016/J.JPEDS.2017.09.043.

18. Ancora G, Pomero G, Ferrari F. Raccomandazioni Per L 'Assistenza Al Neonato Con Ipotermico. 2012.

19. Azzopardi DV, Strohm B, Edwards AD, et al. Moderate Hypothermia to Treat Perinatal Asphyxial Encephalopathy. N Engl J Med. 2009;361(14):1349-58. doi:10.1056/NEJMoa0900854. 
20. Chow S-C, Shao J, Wang H. Sample Size Calculations in Clinical Research.; 2008. doi:10.1097/00000542-200210000-00050.

\section{Supplementary Files}

This is a list of supplementary files associated with this preprint. Click to download.

- NEWSUPPLEMENTARY1SPIRITchecklist.pdf

- SUPPLEMENTARY2CONSENTEnglishversion.docx

- SUPPLEMENTARY3TIMESCHEDULE.pdf 